This is the peer reviewed version of the following article: Prokić MD, Petrović TG, Despotović SG, Vučić T, Gavrić JP, Radovanović TB, Gavrilović BR. The effect of shortterm fasting on the oxidative status of larvae of crested newt species and their hybrids. Comp Biochem Physiol Part A Mol Integr Physiol. 2021;251:110819.

https://doi.org/10.1016/j.cbpa.2020.110819

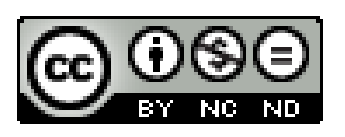

(C) 2020 Elsevier Inc. 


\section{The effect of short-term fasting on the oxidative status of larvae of crested newt species}

and their hybrids

Marko D. Prokić ${ }^{1, *, \#}$, Tamara G. Petrović ${ }^{1, \#}$, Svetlana G. Despotović ${ }^{1}$, Tijana Vučić 2,3 , Jelena P. Gavrić ${ }^{1}$, Tijana B. Radovanović ${ }^{1}$, Branka R. Gavrilović ${ }^{1}$

${ }^{1}$ Department of Physiology, Institute for Biological Research "Siniša Stanković", University of Belgrade, Bulevar despota Stefana 142, 11060 Belgrade, Serbia

2Department of Evolutionary Biology, Institute for Biological Research “Siniša Stanković”, University of Belgrade, Belgrade, Serbia

${ }^{3}$ Faculty of Biology, Institute for Zoology, University of Belgrade, Studentski trg 16, 11000 Belgrade, Serbia

\# authors with equal contribution

* corresponding author:

Marko D. Prokić, Research Associate

Department of Physiology,

Institute for Biological Research "Siniša Stanković", University of Belgrade,

Bulevar despota Stefana 142,

11060 Belgrade, Serbia

Tel.: +381 112078 341; Fax: +381 112761433.

E-mail address: marko.prokic@ibiss.bg.ac.rs (Marko D. Prokić). 


\begin{abstract}
In nature, animals often face periods without food caused by seasonal fluctuations and/or prey scarcity. An organism's physiological response to imposed energetic limitations is followed by changes in mitochondrial functioning (adjustment of energy metabolism) and a reduction of non-essential processes. However, this energy-saving strategy can have its costs. In this study, we examined oxidative stress as one of the possible physiological costs of shortterm, two-week-long food deprivation on developing amphibian larvae of the crested newts Triturus macedonicus and Triturus ivanbureschi and their hybrids. We investigated whether this exogenous factor additionally affected the oxidative status (fitness-related trait) of hybrid individuals. The fasting treatment led to lower growth and a lower body mass and body condition index of individuals. The results revealed that the antioxidant system (AOS) of food-deprived larvae could not cope in a proper manner with reactive oxygen species production under limited energy availability, leading to higher lipid oxidative damage. The lowest AOS response was observed for $\mathrm{H}_{2} \mathrm{O}_{2}$ scavenging parameters (catalase, glutathione peroxidase, and total glutathione), which together with the elevated activity of superoxide dismutase suggested increased $\mathrm{H}_{2} \mathrm{O}_{2}$ concentrations. Comparison between parental species and their hybrids showed that hybrid individuals suffered greater oxidative damage (as demonstrated by higher concentrations of lipid peroxides), indicating that they were more susceptible to fasting-induced oxidative stress. Overall, this study illustrates that: (i) an oxidative event is one of the costs amphibian larvae face during short-term periods of fasting, (ii) hybrids are less capable of dealing with this stressful condition, which can lower their chances of survival in a changing environment.
\end{abstract}

Keywords: antioxidant system; oxidative stress; hybridization; fitness; food deprivation; amphibians; larvae 


\section{Introduction}

All processes of life, even the most basic ones, have an energy cost (Porter and Gates, 1969). An animal's energy and the constitutive compounds required for normal functioning are obtained through food ingestion (McCue, 2010). Although food is essential for development, growth, self-maintenance and reproduction, individuals are often challenged by the absence of food in nature (McCue, 2012). For most amphibian species, periods of intermittent food supply are the result of bouts of foraging in stochastic environments during seasonal fluctuations (Milanovich and Maerz, 2013). Most of the data on this topic in amphibians are focused on prolonged food deprivation during hibernation and/or estivation in adults (Storey, 2002; Secor, 2005; Moreira et al., 2020). However, short-term fasting during the larval period has been studied to a lesser extent than in adults, even though it is commonly seen in the wild (Crespi and Denver, 2005). In larvae, food deprivation can have a negative effect on the duration of the developmental period and individual survival (Denoël and Poncin, 2001). Harsh conditions at early stages might have lasting consequences on the life history of individuals during later life (Scott et al., 2007).

To overcome the energetic limitations imposed by fasting, organisms have developed mechanisms to mobilize internal energy stores, adjust energy metabolism and downsize nonessential processes (Secor and Carey, 2016). Maintaining the biological system under fasting conditions carries hidden costs. The acquisition of sufficient energy in larvae (necessary for growth and maintenance of homeostasis on one hand and the establishment of lipid stores on the other, during periods of food absence) can be followed by molecular damage (Kirschman et al., 2017; Burraco et al., 2020). One of the potential costs of fasting is oxidative stress, mainly because of the metabolic changes caused by modifications to the mitochondria, the major site of reactive oxygen species (ROS) production (Salin et al., 2018), but also because of the activation of the hypothalamic-pituitary-adrenal/interrenal (HPA/I) axis (Crespi and Denver, 2005), and alterations in prooxidant/antioxidant balance (Sorensen et al., 2006; Vázquez-Medina et al., 2010). An increase in ROS levels (especially $\mathrm{H}_{2} \mathrm{O}_{2}$ ) as the result of the energy-saving strategy for fasting has been reported for different groups of organisms (Sorensen et al., 2006; Sharma et al., 2011; Zhang et al., 2013; Salin et al., 2018). Increased ROS levels are usually accompanied by the response of the antioxidant system (AOS), which is equipped with enzymatic and non-enzymatic components (Costantini, 2014). The first line of this defense consists of enzymes (superoxide dismutase - SOD, catalase - CAT, and glutathione peroxidase - GSH-Px) that neutralize ROS $\left(\mathrm{O}_{2}{ }^{-}, \mathrm{H}_{2} \mathrm{O}_{2}\right)$. The second line of the defense comprises the scavenging non-enzymatic antioxidants (glutathione - GSH, sulfhydryl 
(SH) groups, uric acid, vitamins $\mathrm{C}$ and $\mathrm{E}$ ), that remove free radicals, inhibiting the initiation and propagation reactions. Beside GSH, the GSH system includes glutathione reductase (GR) that reduces the oxidized form of glutathione, and the biotransformation enzyme glutathione S-transferase (GST) that participates in the conjugation of GSH with various substances (Halliwell and Gutteridge, 2015). Studies showed that fasting state can increase (VázquezMedina et al., 2011; Schull et al., 2016) or decrease (Morales et al., 2004; Sorensen et al., 2006; Rocha et al., 2008) activities/concentrations of AOS components, depending on various factors (species, tissue, duration of food deprivation). However, the state of oxidative stress occurs only in cases when the produced ROS overwhelms the capacities of the AOS (Halliwell and Gutteridge, 2015).

For the examination of possible oxidative costs of fasting on amphibian larvae we used crested newts, Triturus macedonicus and T. ivanbureschi, and their hybrids as a model organism. We monitored the parameters of the AOS (SOD, CAT, GSH-Px, GSH, GR, GST, and SH groups), oxidative damage (lipid peroxidation (LPO)), and body size (snout-vent length (SVL) and body mass (BM)) after two weeks of food deprivation. The ability of $T$. macedonicus and T. ivanbureschi to hybridize in nature and produce fertile hybrids (Wielstra and Arntzen, 2012; Wielstra et al., 2017; Arntzen et al., 2018), allowed us to also examine the possible effects of food deprivation as an exogenous factor on hybrid fitness. Hybrid individuals often have lower fitness in comparison to parental species due to the effects of endogenous and/or exogenous factors (Burke and Arnold, 2001). In hybrid individuals of crested newts, mitonuclear mismatch (endogenous factor) was marked as one of the main reasons for the increased metabolic rate as compared to parental species (Gvoždík, 2012). Our previous study showed that $\mathrm{F}_{1}$ hybrid individuals of crested newts (of T. macedonicus and T. ivanbureschi) had higher values of antioxidant parameters and exhibited a less integrated AOS in comparison to parental species under non-stressful conditions, indicating the trend of decreased fitness due to endogenous factors (Prokić et al., 2018a). The degree of effects of parental incompatibilities on hybrid fitness can also depend on the environmental (exogenous) factors in which hybrids occur (Stolzenberg et al., 2009; Havird et al., 2019). We believe that following oxidative stress parameters in hybrid larvae that develop under a challenging environment would provide insight into the hybridization phenomenon in natural populations.

Based on the findings that fasting can alter mitochondrial function, induce a significant increase in ROS levels, and activate the HPA/I axis, we assumed that the energy-limited state will: (1) alter the AOS, especially the $\mathrm{H}_{2} \mathrm{O}_{2}$-scavenging part of the system (CAT, GSH-Px 
and $\mathrm{GSH}$ ) due to the increase in mitochondrial $\mathrm{H}_{2} \mathrm{O}_{2}$ levels; (2) cause oxidative stress in crested newt larvae in both parental species and hybrids. If a higher investment in the maintenance of oxidative balance due to parental incompatibilities (mitonuclear mismatch) occurs in hybrids, we expect that (3) periods of fasting will lead to higher levels of oxidative damage in hybrids in relation to the parental species.

\section{Materials and Methods}

\subsection{Experimental design}

Triturus ivanbureschi and T. macedonicus adults (the parental generation) were collected from natural populations in 2014 and 2015, respectively. The capture of animals for the experiment was approved by the Ministry of Energy, Development and Environmental Protection of the Republic of Serbia (Permit No. 353-01-75/2014-08), and the Environmental Protection Agency of Montenegro (Permit No. UPI-328/4). The experimental procedure was approved by the Animal Ethical Committee of the Institute for Biological Research "Siniša Stanković”, University of Belgrade (Decision No. 03-03/16). For the present experiment, the animals were crossed at the beginning of March 2018, after hibernation in the cold chamber at a constant temperature $\left(4^{\circ} \mathrm{C}\right)$. Crossings between parental species were performed in seminatural conditions (large, 500-L containers were filled with water, closed with a protective net, containing plastic strips as underwater vegetation for egg deposition, bricks for shelter and plastic floating islets). Four different crossings were made to obtain larvae of the species and their reciprocal $F_{1}$ hybrids, as follows: 1) T. ivanbureschi (T. ivanbureschi $6 q \times 3 \AA$ ), 2) T. macedonicus (T. macedonicus $3 q \times 3 \hat{O}$ ), 3) T. ivanbureschi-mothered hybrids (3 T. ivanbureschi $q \times 3$ T. macedonicus $\left.{ }^{\Uparrow}\right)$, and 4) T. macedonicus-mothered hybrids (2 T. macedonicus $\uparrow \times 2$ T. ivanbureschi $\precsim$ ).

When females started depositing eggs, they were transferred to separate $10-\mathrm{L}$ aquaria, half-filled with dechlorinated tap water. Plastic strips were set for egg deposition. Eggs were collected daily and raised in Petri dishes. After hatching, the larvae were raised in 100-mL plastic cups. When larvae reached stage 50 (beginning of the formation of hind limbs; Glücksohn, 1932), they were transferred to 2-L plastic containers (one larva per container) half-filled with dechlorinated tap water. The water in the Petri dishes, cups and containers was changed every other day. To minimize potential environmental effects, all individuals were reared in the same controlled laboratory conditions under an ambient photoperiod. The temperature was maintained at $19^{\circ} \mathrm{C}$. During the earlier stages, the larvae were fed ad libitum 
with Artemia sp., and at later stages with Tubifex sp. At stage 62 (fully developed digits, limbs and tail; Glücksohn, 1932) the larvae only increased in size until the end of metamorphosis. At this stage, the larvae were randomly divided into fasting and control groups. The number of individuals in the fasting group was as follows: for T. ivanbureschi= 13, $T$. macedonicus $=15, T$. ivanbureschi-mothered hybrid $=13$ and $T$. macedonicusmothered hybrid $=15$. The number of individuals in the control group was as follows: for $T$. ivanbureschi $=8, T$. macedonicus $=10, T$. ivanbureschi-mothered hybrid $=9$ and $T$. macedonicus-mothered hybrid $=15$. Larvae were kept individually in 2-L plastic containers (one larva per container), to avoid intraspecific aggression and cannibalism, and for comparison of biometric parameters of individuals at the beginning and end of the experiment. Individuals in the control group were maintained at the food ratio they were exposed to earlier. Twice a day, feces were removed from the containers to minimize possible feces ingestion and to keep the water clean. Water in containers was changed every other day. Individuals in the fasting group were maintained on the fasting treatment for 14 days, which is a credible period of short-term food deprivation that might be experienced by newts in nature (Irwin et al., 1999; Milanovich and Maerz, 2013). All individuals survived until the end of the experiment. The larvae were killed by immersion in liquid nitrogen and were kept at $-80{ }^{\circ} \mathrm{C}$ until further analyses (Underwood et al., 2013).

The body mass and SVL of individuals were measured at the start and end of the experiment. For determination of SVL, we used photographs of the dorsal view (from the tip of the snout to the level of the posterior edge of hind legs) of the larvae with a Sony DSCF828 digital camera (24-bit color and $3264 \times 2448$ pixel resolution, MP; Sony Corp., Tokyo, Japan), and ImageJ software (Rasband, 2019). The body condition index (CI) was calculated on log-transformed data for BM and SVL according to Labocha et al. (2014).

\subsection{Sample processing and biochemical analyses}

The whole body of larvae was minced and mixed to obtain material as homogenous as possible. About $0.2 \mathrm{~g}$ of mixed tissues were taken for LPO determination while the rest was used for other biochemical analyses. Analysis of the level of LPO included homogenization and sonication in 10 volumes of an ice-cold Tris-HCl buffer (pH 7.4), followed by centrifugation at $10,000 \times \mathrm{g}$ for $10 \mathrm{~min}$ at $4^{\circ} \mathrm{C}$ in $40 \%$ trichloroacetic acid (TCA) to obtain the supernatant. For measurement of antioxidant parameters, the tissue was homogenized for $45 \mathrm{~s}$ in 5 volumes of an ice-cold $25 \mathrm{mM}$ sucrose buffer (pH 7.4) containing $10 \mathrm{mM}$ Tris- $\mathrm{HCl}$ and 5 mM EDTA, with an Ultra Turrax homogenizer (T-18, IKA-Werk, Germany). The 
homogenates were then sonicated at $10 \mathrm{kHz}$ for $30 \mathrm{~s}$ on ice (Takada et al., 1982) using a Sonopuls ultrasonic homogenizer (HD 2070, Bandelin Electronic, Germany). For the determination of GSH concentration, the sonicates were centrifuged at 5,000 $\times \mathrm{g}$ for $10 \mathrm{~min}$ in $10 \%$ sulfosalicylic acid and the protein precipitate was discarded. For measurement of the other AOS parameters, the sonicates were centrifuged at $100,000 \times \mathrm{g}$ for $90 \mathrm{~min}$ at $4{ }^{\circ} \mathrm{C}$ to obtain the supernatant.

The protein content was determined at $500 \mathrm{~nm}$ using the Lowry method (Lowry et al., 1951) with bovine serum albumin (BSA) as standard. SOD activity was measured at $480 \mathrm{~nm}$ according to the assay described by Misra and Fridovich (1972) that is based on the ability of SOD to inhibit the autoxidation of epinephrine. The Claiborne (1984) method for CAT analysis, which includes measurement of hydrogen peroxide degradation at $240 \mathrm{~nm}$, was performed. GSH-Px activity was measured according to the protocol outlined by Tamura et al. (1982); to estimate GR activity we used the assay of Glatzle et al. (1974). According to both methods, the activity is based on the rate of NADPH oxidation. GST activity towards 1chloro-2,4-dinitrobenzene (CDNB) was determined using the protocol of Habig et al. (1974). GSH-dependent enzymes (GSH-Px, GR, and GST) were measured at $340 \mathrm{~nm}$. The activities of all antioxidant enzymes were expressed in U/mg protein. GSH concentration was determined according to the 5,5'-dithio-bis-(2-nitrobenzoic acid) (DTNB) enzymatic recycling method (Griffith, 1980) and was expressed in nmol/g tissue. The method of Ellman (1959) was used for the estimation of protein sulfhydryl (-SH) group concentrations, expressed as $\mu \mathrm{mol} / \mathrm{mg}$ protein. Non-enzymatic parameters (GSH and protein -SH groups) were measured at $412 \mathrm{~nm}$. For the measurement of LPO, we used the thiobarbituric acidreactive substance (TBARS) assay of Rehncrona et al. (1980). Lipid peroxide concentrations were evaluated at $532 \mathrm{~nm}$ and expressed in nmol/mg tissues. All biochemical measurements were performed at $19^{\circ} \mathrm{C}$ with a Shimadzu UV $1800 \mathrm{UV}-\mathrm{VIS}$, (Shimadzu, Japan) spectrophotometer with a temperature-controlled cuvette holder. More details for the biochemical measurements are presented in the Supplementary Material.

\subsection{Statistical analyses}

Possible outliers were checked by Grubb’s test. Assumptions of normality (Kolmogorov-Smirnov test) and homogeneity of variances (Levine’s test) were respected. Possible differences between two independent variables: "species” (parental species and hybrids), treatments (fasting and control) and their interaction for biometric and oxidative stress parameters, were analyzed using factorial ANOVA. On parameters for which a 
significant interaction between factors (treatment x species) was reported, we performed pairwise multiple comparisons - Tukey’s test. For parameters with significant differences for each factor, we applied the post hoc Tukey HSD test to determine further differences between the parameters. In the analysis of biometric parameters recorded at the end of the experiment, we included the initial values as covariate in the ANCOVA test. The criterion for significance was set as $\mathrm{p} \leq 0.05$. Statistical analyses were performed using STATISTICA 8.0 (StatSoft, Inc., 2007), with the exception of pairwise multiple comparisons, which were calculated in XLSTAT, Ver. 2014.5.03 (Addinsoft, 2015).

\section{Results}

\subsection{Biometric parameters}

Biometric parameters (SVL, BM and CI) for all examined groups are given in Table 1. At the beginning of the experiment, we observed significant differences only between species (Table 2). At the end of the experiment, we observed differences after the treatments for all parameters and for the BM between species (Table 2). No significant interactions between factors were detected (Table 2). Two-week fasting individuals of all groups had significantly lower values for BM, SVL and CI in comparison to control individuals at the end of the treatment (T. macedonicus SVL- F=24.8, $\mathrm{p}<0.0001, \mathrm{df}=1, \mathrm{BM}-\mathrm{F}=52.0, \mathrm{p}<0.0001$, $\mathrm{df}=1, \mathrm{CI}-\mathrm{F}=41.4, \mathrm{p}<0.0001, \mathrm{df}=1 ;$ T. ivanbureschi $\mathrm{SVL}-\mathrm{F}=47.6, \mathrm{p}<0.0001, \mathrm{df}=1, \mathrm{BM}-$ $\mathrm{F}=59.1, \mathrm{p}<0.0001, \mathrm{df}=1, \mathrm{CI}-\mathrm{F}=37.2, \mathrm{p}<0.0001 ;$ T. macedonicus mothered hybrids: SVL$\mathrm{F}=39.0, \mathrm{p}<0.0001, \mathrm{df}=1, \mathrm{BM}-\mathrm{F}=39.2, \mathrm{p}<0.0001, \mathrm{df}=1, \mathrm{CI}-\mathrm{F}=34.6, \mathrm{p}<0.0001, \mathrm{df}=1 ; T$. ivanbureschi mothered hybrid SVL- F=40.9, $\mathrm{p}<0.0001$, $\mathrm{df}=1, \mathrm{BM}-\mathrm{F}=70.7, \mathrm{p}<0.0001, \mathrm{df}=1$, CI- F=53.9, $\mathrm{p}<0.0001, \mathrm{df}=1)$.

\subsection{Oxidative stress parameters}

The main aim of this study was to determine the effects of fasting on crested newt larvae (control vs fasting), and possible differences between the examined species in response to fasting; thus, we will present only these results. The rest of the results (such as differences between species in the control group) can be found in the Supplementary Material (Tables S1 and S2). The results for factorial ANOVA showed significant differences for all examined parameters for the factors species and treatment, the exception being GR, where a significant difference was detected only between the species (Table 3). Significant interactions for treatment x species were observed for SOD, GSH-Px, SH groups and LPO (Table 3). All possible combinations of the post hoc test on parameters with significant interactions are shown in Table S2. Post hoc analysis of the interaction between species and treatment for 
SOD showed that fasting individuals of both parental species (T. macedonicus and $T$. ivanbureschi) had higher values than those of the control; also, SOD activity was higher in the parental species in comparison to the hybrids (T. macedonicus-mothered and $T$. ivanbureschi-mothered hybrid) under fasting treatment (Fig. 1 and Table S2). The GSH-Px value was lower in the fasting group of T. macedonicus, $T$. macedonicus-mothered and $T$. ivanbureschi-mothered hybrid in comparison to matching controls (Fig. 1 and Table S2). Comparison under fasting treatments revealed that T. ivanbureschi and T. macedonicusmothered hybrid had higher activities of GSH-Px than T. macedonicus and the $T$. ivanbureschi-mothered hybrid (Fig. 1 and Table S2). For the interactions of SH groups, we observed a lower concentration in fasting individuals of T. macedonicus and T. ivanbureschimothered hybrid in comparison to matching controls, and as regards differences between species under the fasting regime, SH groups were higher in $T$. ivanbureschi and the $T$. macedonicus-mothered hybrid than in the other two species (Fig. 2 and Table S2). Comparison of LPO values showed that fasting larvae of all examined groups had higher values than the control (Fig. 2 and Table S4). Fasting hybrid individuals had a higher concentration of LPO than individuals of fasting parental species, T. ivanbureschi-mothered hybrid than both parents, while the T. macedonicus-mothered hybrid had a higher concentration of LPO than only T. macedonicus (Fig. 2 and Table S2). For parameters with significant differences for the factors species and treatment (CAT, GSH and GST), post hoc tests were performed for the treatments (control vs fasting) and species (all species under fasting treatment); interspecies differences under non-stressful (control) conditions were of interest in our previously published study (Prokić et al., 2018a). CAT activity was lower in fasting individuals of T. macedonicus, T. ivanbureschi and T. ivanbureschi-mothered hybrid as compared to the control (Fig. 1). Comparison between species in fasting groups showed that $T$. ivanbureschi individuals had the highest value for CAT activity. Catalase activity was also higher in T. macedonicus and the T. macedonicus-mothered hybrid in comparison to the T. ivanbureschi-mothered hybrid (Fig. 1). Fasting lowered GSH concentration in all examined species, while comparison between species revealed that GSH was lowest in $T$. macedonicus (Fig. 1). Significant differences in GST activity between fasting and control individuals were reported for T. ivanbureschi and T. macedonicus-mothered hybrid. The fasting larvae of T. ivanbureschi also had higher GST than all other groups under the fasting treatment (Fig. 1). GR was the only parameter with significant differences only for the factor species, however, the post hoc test showed significant differences only between control individuals (Table S1). 


\section{Discussion}

When the balance between energy consumption and expenditure is disrupted and the energetic conditions decline, organisms are forced to induce shifts in energy resource allocation, diverting them away from different functions such as growth, reproduction, immune and antioxidant defenses (De Block and Stoks, 2008; Monaghan et al., 2009; Isaksson et al., 2011; Schull et al., 2016; Prokić et al., 2018b; Prokić et al., 2019). Two weeks of fasting led to body-mass loss and lower growth (body length), affecting the body condition of crested newt larvae. The observed lower CI indicates a lower fat content that together with non-optimal growth conditions in larvae can further alter the development of new structures and metamorphosis (Metcalfe and Monaghan, 2001). Even though we did not follow post fasting effects, in the literature a smaller body size of amphibian larvae either results in a smaller size of metamorphs or it can lead to an increase in 'catch-up' growth to compensate for the adverse effects of fasting. Both possible scenarios can have negative effects on different fitness traits of juveniles (locomotor abilities, metabolic rates, endurance, resistance to desiccation and feeding success, immune function) (Semlitsch et al., 1988; Beck and Congdon, 2000; Gervasi and Foufopoulos, 2008; Burraco et al., 2017, 2020; Székely et al., 2020).

The newt larvae are very sensitive to different perturbations of environmental conditions (different temperatures, permanency of the ponds, etc.)(Denoël and Poncin, 2001). Short-term fasting is among the most common negative factors, caused by different environmental perturbations. In this study, we assumed that short-term fasting affects the oxidative status of the developing larvae of crested newts. The prediction was based on the finding that a two-week fasting period in ectotherms (brown trout Salmo trutta) induced a significant increase in hepatic mitochondrial $\mathrm{H}_{2} \mathrm{O}_{2}$ levels (Salin et al., 2018), and the activation of the HPI axis in amphibian larvae (an increase in corticosterone and corticotropin-releasing factor) in response to food unavailability (Crespi and Denver, 2005). Herein, we observed a lower antioxidant defense and higher oxidative damage (LPO concentration) in fasting larvae of crested newts in comparison to controls. The only AOS parameter that was higher was SOD (result seen in both parental species). The corresponding increase in SOD is expected due to an increased mitochondrial superoxide radical leak (Schull et al., 2016). Activation of SOD causes increased formation of intracellular $\mathrm{H}_{2} \mathrm{O}_{2}$ (Halliwell and Gutteridge, 2015). However, the two subsequently-activated components in this biochemical cascade, the $\mathrm{H}_{2} \mathrm{O}_{2}$-scavenging enzymes, failed. The absence of an adequate 
response from CAT and GSH-Px in removing $\mathrm{H}_{2} \mathrm{O}_{2}$ is in agreement with the significant increases in $\mathrm{H}_{2} \mathrm{O}_{2}$ concentrations that are observed during fasting in the mitochondria of different animals (Sorensen et al., 2006; Salin et al., 2018; Roussel et al., 2019). Aside from direct effects, such as oxidative damage of biomolecules, it was suggested that increased $\mathrm{H}_{2} \mathrm{O}_{2}$ concentration as a secondary messenger could play a role in the re-feeding signal (Sylvie et al., 2012) by modulating cellular hormesis (Costantini, 2014; Schull et al., 2016). A decrease was also observed in the concentration of GSH, an important component of the second line of defense. GSH is crucial for the adaptive response against oxidative stress and a variety of ROS (Halliwell and Gutteridge, 2015). It is a potent reductant and cofactor for GSH-Px and GST, and is involved in the removal of hydrogen peroxide and lipid hydroperoxides, the main targets for the GSH redox system (Forman et al., 2009). Lower concentrations of GSH in fasting larvae can further contribute to increased concentrations of $\mathrm{H}_{2} \mathrm{O}_{2}$ and the accumulation of oxidative damage. Lower thiol concentrations were also reported in T. macedonicus and T. ivanbureschi-mothered hybrid under limited food availability, suggesting further alterations in the redox status. Higher GST activity in $T$. ivanbureschi and T. macedonicus-mothered hybrids could be the result of the activation of the detoxification system that prevents lipid hydroperoxide formation. Increased generation of lipid peroxides was reported during both short- and long-term fasting and in ectho- and endotherms (Ogasawara et al., 1989; Pascual et al., 2003; Morales et al., 2004; Sorensen et al., 2006; Furné et al., 2009; Sinha et al., 2015; Varju et al., 2018). Fasting in animals, aside from increasing mitochondrial ROS production, also affects lipid metabolism, leading to high fatty acid unsaturation (higher content of unsaturated fatty acids rich in double bonds) in the cell membranes making them more prone to oxidative damage (Sorensen et al., 2006). Moreover, changes in the lipid composition of the mitochondrial membrane can further modify mitochondrial $\mathrm{H}_{2} \mathrm{O}_{2}$ generation (Ramsey et al., 2005).

The oxidative stress during food deprivation could be interpreted using resource- or energy-based models (Costantini, 2019). Limited-energy conditions can result in the inability of an organism to increase its antioxidant defense towards increased ROS production. Maintaining the AOS and repair systems requires the consumption of energy (Monaghan et al., 2009). It was shown that in the absence of food (energy), the animals were incapable of maintaining high levels of AOS activity (Costantini and Møller, 2008; De Block and Stoks, 2008; Isaksson et al., 2011). Studies on poikilothermic organisms suggest that food deprivation can cause an impairment of the glutathione redox system (Furné et al., 2009), and significantly lower the concentration of GSH (Pascual et al., 2003; Sinha et al., 2015; Varju 
et al., 2018) There is evidence that nutritional restriction negatively affects GSH metabolism, due to a deficiency of the cysteine-precursor amino acids used for GSH synthesis, which are obtained directly from food or by the metabolism of dietary methionine (Shimizu and Morita, 1992; Paterson et al., 2001). This may also produce a resource-allocation conflict between cellular GSH levels and the synthesis of proteins containing cysteine (de Magalhães and Church, 2006). Moreover, the enzymatic activity of GSH-Px depends on the presence of the essential dietary micronutrient selenium (Kumar et al., 2009; Isaksson et al., 2011). Newt larvae during the period of fasting continue to grow, but at a lower rate in comparison to controls. However, the energy used for somatic growth can additionally deviate energy from the AOS (Monaghan et al., 2009).

Two weeks of fasting revealed that hybrid individuals suffered greater oxidative damage than the parental species (evidenced by the increase in LPO concentration) $-T$. ivanbureschi-mothered hybrid in comparison to both parents, and the T. macedonicusmothered hybrid in comparison to T. macedonicus. Hybrid individuals also displayed lower SOD activity. The more pronounced effects of fasting in hybrid individuals can be the result of the greater amount of energy they need to invest in the maintenance of physiological processes (standard metabolic rates and the AOS) (Gvoždík, 2012; Prokić et al., 2018a; Petrović et al., 2020). Previous observations on hybrid individuals of crested newts showed that they displayed an elevated AOS, were more active and aggressive, and were larger in body size in comparison to parental species under non-stressed conditions (Prokić et al., 2018a; Petrović et al., 2020). The availability of food resources was found to be crucial in limiting Triturus newt hybrids’ endurance in the environment because of the greater expenditure of energy in the maintenance of high metabolic rates caused by mitonuclear mismatch (Gvoždík, 2012). A higher oxidative stress can be observed as a physiological constraint affecting fitness-related traits and mediating the life-history of hybrids through the progressive deterioration of tissues. The limited capacity to cope with exogenous factors and stress in a period of food deprivation could affect the survival of hybrid individuals in natural populations and could contribute to relatively narrow geographical hybrid zones in Triturus newts.

\section{Conclusion}

Even short-term food deprivation in crested newt larvae can lead to an oxidative event that is followed by a lower response of the AOS. The effects of the observed oxidative costs on the short- and long-term life histories (metamorphosis, growth patterns, reproductive 
performance, aging and survival) of newts still require investigation. Comparisons between parental species and hybrids revealed more pronounced oxidative damage in hybrid individuals. The results from this study should be useful in our efforts to gain a better understanding of the possible role of oxidative stress in animals facing limited energy conditions, as well as in ecologically-mediated hybrid inferiority.

\section{Acknowledgements}

This study was supported by the Ministry of Education, Science and Technological Development of the Republic of Serbia, Contract No. 451-03-68/2020-14/200007. The authors are grateful to Dr. Goran Poznanović for proofreading the manuscript, to Sonja Nikolić and Sanja Šajkunić for technical assistance during experiment realization and anonymous reviewers for constructive suggestions that improved our work.

\section{Credit author statement:}

Marko D. Prokić: Conceptualization, Formal analysis, Investigation, Data Curation, Writing - Original Draft, Visualization, Supervision, Project administration; Tamara G. Petrović: Conceptualization, Investigation, Writing - Original Draft, Visualization; Svetlana G. Despotović: Investigation, Writing - Review \& Editing, Tijana Vučić: Investigation, Resources, Writing - Review \& Editing; Jelena P. Gavrić: Investigation, Writing - Review \& Editing; Tijana B. Radovanović: Investigation, Writing - Review \& Editing; Branka R. Gavrilović: Investigation, Writing - Review \& Editing; 


\section{References}

Addinsoft, XLSTAT 2015. Data Analysis and Statistics Software for Microsoft Excel. Addinsoft: Paris, France.

Arntzen, J.W., Üzüm, N., Ajduković, M.D., Ivanović, A., Wielstra, B., 2018. Absence of heterosis in hybrid crested newts. PeerJ 6, e5317.

Beck, C.W., Congdon, J.D., 2000. Effects of age and size at metamorphosis on performance and metabolic rates of southern toad, Bufo terrestris, metamorphs. Funct. Ecol. 14, 3238.

Burke, J.M., Arnold, M.L., 2001. Genetics and the fitness of hybrids. Annu. Rev. Genet. 35, 31-52.

Burraco, P., Díaz-Paniagua, C., Gomez-Mestre, I., 2017. Different effects of accelerated development and enhanced growth on oxidative stress and telomere shortening in amphibian larvae. Sci. Rep. 7, 1-11.

Burraco, P., Valdés, A.E., Orizaola, G., 2020. Metabolic costs of altered growth trajectories across life transitions in amphibians. J. Anim. Ecol. 89, 855-866.

Claiborne, A., 1984. Catalase activity. In: Greenwald, R.A. (Ed.), Handbook of Methods for Oxygen Radical Research. CRC Press Inc., Boca Raton, pp. 283-284.

Costantini, D., 2014. Oxidative Stress and Hormesis in Evolutionary Ecology and Physiology. A Marriage Between Mechanistic and Evolutionay Approaches. New York: Springer.

Costantini, D., 2019. Understanding diversity in oxidative status and oxidative stress: the opportunities and challenges ahead. J. Exp. Biol. 222, jeb194688.

Costantini, D., Møller, A.P., 2008. Carotenoids are minor antioxidants for birds. Funct. Ecol. 22, 367-370.

Crespi, E.J., Denver, R.J., 2005. Roles of stress hormones in food intake regulation in anuran amphibians throughout the life cycle. Comp. Biochem. Physiol. Part A Mol. Integr. $141,381-390$.

De Block, M., Stoks, R., 2008. Compensatory growth and oxidative stress in a damselfly. Proc. R. Soc. B 275, 781-785.

de Magalhães, J.P., Church, G.M., 2006. Cells discover fire: Employing reactive oxygen species in development and consequences for aging. Exp. Gerontol. 41, 1-10.

Denoël, M., Poncin, P., 2001. The effect of food on growth and metamorphosis of paedomorphs in Triturus alpestris apuanus. Arch. Hydrobiol. 152, 661-670.

Ellman, G.L., 1959. Tissue sulfhydryl groups. Arch. Biochem. Biophys. 82, 70-77. 
Forman, H.J., Zhang, H., Rinna, A., 2009. Glutathione: overview of its protective roles, measurement, and biosynthesis. Mol. Aspects Med. 30, 1-12.

Furné, M., García-Gallego, M., Hidalgo, M.C., Morales, A.E., Domezain, A., Domezain, J., Sanz, A., 2009. Oxidative stress parameters during starvation and refeeding periods in Adriatic sturgeon (Acipenser naccarii) and rainbow trout (Oncorhynchus mykiss). Aquacult. Nutr. 15, 587-595.

Gervasi, S.S., Foufopoulos, J., 2008. Costs of plasticity: responses to desiccation decrease postmetamorphic immune function in a pond-breeding amphibian. Funct. Ecol. 22, 100-108.

Glatzle, D., Vuilleumier, J.P., Weber, F., Decker, K., 1974. Glutathione reductase test with whole blood, a convenient procedure for the assessment of the riboflavin status in humans. Experientia 30, 665-667.

Glücksohn, S., 1932. Äußere Entwicklung der Extremitäten und Stadieneinteilung der Larvenperiode von Triton taeniatus Leyd. und Triton cristatus Laur. Wilhelm Roux' Arch. Entwickl.-Mech. Org. 125, 341-405. doi:10.1007/BF00576359

Griffith, O.W., 1980. Determination of glutathione and glutathione disulfide using glutathione reductase and 2-vinylpyridine. Anal. Biochem. 106, 207-212.

Gvoždík, L., 2012. Metabolic costs of hybridization in newts. Folia Zool. 61, 197-201. Habig, W.H., Pabst, M.J., Jakoby, W.B., 1974. Glutathione S-transferases. The first enzymatic step in mercapturic acid formation. J. Biol. Chem. 249, 7130-7139.

Halliwell, B., Gutteridge, J.M., 2015. Free Radicals in Biology and Medicine 4th edition. Oxford University Press, USA.

Havird, J.C., Weaver, R.J., Milani, L., Ghiselli, F., Greenway, R., Ramsey, A.J., Jimenez, A.G., Dowling, D.K., Hood, W.R., Montooth, K.L., Estes, S., Schulte, P.M., Sokolova, I.M., Hill, G.E., 2019. Beyond the powerhouse: integrating mitonuclear evolution, physiology, and theory in comparative biology. Integr. Comp. Biol. 59, 856-863.

Irwin, L.N., Talentino, K.A., Caruso, D.A., 1999. Effect of fasting and thermal acclimation on metabolism of juvenile axolotls (Ambystoma mexicanum). Exp. Biol. Online 3, 1-11.

Isaksson, C., Sheldon, B.C., Uller, T., 2011. The challenges of integrating oxidative stress into life-history biology. Bioscience 61, 194-202.

Kirschman, L.J., McCue, M.D., Boyles, J.G., Warne, R.W., 2017. Exogenous stress hormones alter energetic and nutrient costs of development and metamorphosis. J. Exp. Biol. 220, 3391-3397. 
Kumar, N., Garg, A.K., Dass, R.S., Chaturvedi, V.K., Mudgal, V., Varshney, V.P., 2009.

Selenium supplementation influences growth performance, antioxidant status and immune response in lambs. Anim. Feed Sci. Technol. 153, 77-87.

Labocha, M.K., Schutz, H., Hayes, J.P., 2014. Which body condition index is best?. Oikos 123, 111-119.

Lowry, O.H., Rosebrough, N.J., Farr, A.L., Randall, R.J., 1951. Protein measurement with the Folin phenol reagent. J. Biol. Chem. 193, 265-275.

McCue, M.D. Comparative Physiology of Fasting, Starvation, and Food Limitation. New York, Springer, 2012.

McCue, M.D., 2010. Starvation physiology: reviewing the different strategies animals use to survive a common challenge. Comp. Biochem. Physiol. Part A Mol. Integr. 156, 1-18.

Metcalfe, N.B., Monaghan, P., 2001. Compensation for a bad start: grow now, pay later?. Trends Ecol. Evol. 16, 254-260.

Milanovich, J.R., Maerz, J.C., 2013. Realistic fasting does not affect stable isotope levels of a metabolically efficient salamander. J. Herpetol. 47, 544-548.

Misra, H.P., Fridovich, I., 1972. The role of superoxide anion in the autoxidation of epinephrine and simple assay for superoxide dismutase. J. Biol. Chem. 247, 3170-3175.

Monaghan, P., Metcalfe, N.B., Torres, R., 2009. Oxidative stress as a mediator of life history trade-offs: mechanisms, measurements and interpretation. Ecol. Lett. 12, 75-92.

Morales, A.E., Pérez-Jiménez, A., Hidalgo, M.C., Abellán, E., Cardenete, G., 2004. Oxidative stress and antioxidant defenses after prolonged starvation in Dentex dentex liver. Comp. Biochem. Physiol. C Toxicol. Pharmacol. 139, 153-161.

Moreira, D.C., Carvajalino-Fernández, J.M., Silva, W.P., Kuzniewski, F., Navas, C.A., de Carvalho, J.E., Hermes-Lima, M., 2020. Preparation for oxidative stress in Proceratophrys cristiceps (Anura, Odontophrynidae) naturally estivating in the Brazilian Caatinga. Sci. Total Environ. 723, 137957.

Ogasawara, T., Ohnhaus, E.E., Hoensch, H.P., 1989. Glutathione and its related enzymes in the small intestinal mucosa of rats: effects of starvation and diet. Res. Exp. Med. 189, 195-204.

Pascual, P., Pedrajas, J.R., Toribio, F., López-Barea, J., Peinado, J., 2003. Effect of food deprivation on oxidative stress biomarkers in fish (Sparus aurata). Chem.-Biol. Interact. 145, 191-199. 
Paterson, P.G., Lyon, A.W., Kamencic, H., Andersen, L.B., Juurlink, B.H., 2001. Sulfur amino acid deficiency depresses brain glutathione concentration. Nutri. Neurosci. 4, 213-222.

Petrović, T.G., Vučić, T.Z., Nikolić, S.Z., Gavrić, J.P., Despotović, S.G., Gavrilović, B.R., Radovanović, T.B., Faggio, C., Prokić, M.D., 2020. The effect of shelter on oxidative stress and aggressive behavior in crested newt larvae (Triturus spp.). Animals, 10, 603.

Porter, W.P., Gates, D.M., 1969. Thermodynamic equilibria of animals with environment. Ecol. Monogr. 39, 227-244.

Prokić, M.D., Despotović, S.G., Vučić, T.Z., Petrović, T.G., Gavrić, J.P., Gavrilović, B.R., Radovanović, T.B., Saičić, Z.S., 2018a. Oxidative cost of interspecific hybridization: a case study of two Triturus species and their hybrids. J. Exp. Biol. 221, jeb182055.

Prokić, M.D., Gavrić, J.P., Petrović, T.G., Despotović, S.G., Gavrilović, B.R., Radovanović, T.B., Krizmanić, I.I., Pavlović, S.Z., 2019. Oxidative stress in Pelophylax esculentus complex frogs in the wild during transition from aquatic to terrestrial life. Comp. Biochem. Physiol. Part A Mol. Integr. 234, 98-105.

Prokić, M.D., Petrović, T.G., Gavrić, J.P., Despotović, S.G., Gavrilović, B.R., Radovanović, T.B., Faggio, C., Saičić, Z.S., 2018b. Comparative assessment of the antioxidative defense system in subadult and adult anurans: A lesson from the Bufotes viridis toad. Zoology 130, 30-37.

Ramsey, J.J., Harper, M.E., Humble, S.J., Koomson, E.K., Ram, J.J., Bevilacqua, L., Hagopian, K., 2005. Influence of mitochondrial membrane fatty acid composition on proton leak and $\mathrm{H}_{2} \mathrm{O}_{2}$ production in liver. Comp. Biochem. Physiol. B Biochem. Mol. Biol. 140, 99-108.

Rasband, W.S., ImageJ, U. S. National Institutes of Health, Bethesda, Maryland, USA, https://imagej.nih.gov/ij/, 1997-2019.

Rehncrona, S., Smith, D.S., Akesson, B., Westerberg, E., Siesjö, B.K., 1980. Peroxidative changes in brain cortical fatty acids and phospholipids, as characterized during $\mathrm{Fe}^{2+}$ and ascorbic acid stimulated lipid peroxidation in vitro. J. Neurochem. 34, 1630-1638.

Rocha, S.G., Fonseca, A., Rodrigues, M., Dantas, F., Caldeira-de-Araujo, A., Santos, R., 2008. Comet assay to determine DNA damage induced by food deprivation in rats. Acta Biol. Hung. 59, 315-325.

Roussel, D., Boël, M., Mortz, M., Romestaing, C., Duchamp, C., Voituron, Y., 2019. Threshold effect in the $\mathrm{H}_{2} \mathrm{O}_{2}$ production of skeletal muscle mitochondria during fasting and refeeding. J. Exp. Biol. 222, jeb196188. 
Salin, K., Villasevil, E.M., Anderson, G.J., Auer, S.K., Selman, C., Hartley, R.C., Mullen, W., Chinopoulos., C., Metcalfe, N.B., 2018. Decreased mitochondrial metabolic requirements in fasting animals carry an oxidative cost. Funct. Ecol. 32, 2149-2157.

Schull, Q., Viblanc, V.A., Stier, A., Saadaoui, H., Lefol, E., Criscuolo, F., Bizze, P., Robin, J.P., 2016. The oxidative debt of fasting: evidence for short-to medium-term costs of advanced fasting in adult king penguins. J. Exp. Biol. 219, 3284-3293.

Scott, D.E., Casey, E.D., Donovan, M.F., Lynch, T.K., 2007. Amphibian lipid levels at metamorphosis correlate to post-metamorphic terrestrial survival. Oecologia 153, 521532.

Secor, S.M., 2005. Physiological responses to feeding, fasting and estivation for anurans. J. Exp. Biol. 208, 2595-2609.

Secor, S.M., Carey, H.V., 2016. Integrative physiology of fasting. Compr. Physiol. 6, 773825.

Semlitsch, R.D., Scott, D.E., Pechmann, J.H., 1988. Time and size at metamorphosis related to adult fitness in Ambystoma talpoideum. Ecology 69, 184-192.

Sharma, P.K., Agrawal, V., Roy, N., 2011. Mitochondria-mediated hormetic response in life span extension of calorie-restricted Saccharomyces cerevisiae. Age 33, 143-154.

Shimizu, M., Morita, S., 1992. Effects of feeding and fasting on hepatolobular distribution of glutathione and cadmium-induced hepatotoxicity. Toxicology 75, 97-107.

Sinha, A.K., AbdElgawad, H., Zinta, G., Dasan, A.F., Rasoloniriana, R., Asard, H., Blust, R., De Boeck, G., 2015. Nutritional status as the key modulator of antioxidant responses induced by high environmental ammonia and salinity stress in European sea bass (Dicentrarchus labrax). PloS one, 10, e0135091.

Sorensen, M., Sanz, A., Gomez, J., Pamplona, R., Portero-Otin, M., Gredilla, R., Barja, G., 2006. Effects of fasting on oxidative stress in rat liver mitochondria. Free Radic. Res. 40, 339-347.

StatSoft, Inc., 2007. STATISTICA (data analysis software system), version 8.0. www.statsoft.com.

Stolzenberg, N., The, B.N., Salducci, M.D., Cavalli, L., 2009. Influence of environment and mitochondrial heritage on the ecological characteristics of fish in a hybrid zone. PloS one, 4, e5962.

Storey, K.B., 2002. Life in the slow lane: molecular mechanisms of estivation. Comp. Biochem. Physiol. Part A Mol. Integr. 133, 733-754. 
Sylvie, G., Marion, K., Yvon, L.M., Jean-Patrice, R., Criscuolo, F., 2012. Of the importance of metabolic phases in the understanding of oxidative stress in prolonged fasting and refeeding. Physiol. Biochem. Zool. 85, 415-420.

Székely, D., Cogălniceanu, D., Székely, P., Armijos-Ojeda, D., Espinosa-Mogrovejo, V., Denoël, M., 2020. How to recover from a bad start: size at metamorphosis affects growth and survival in a tropical amphibian. BMC Ecol. 20, 1-8.

Takada, Y., Noguchit, T., Kayiyama, M., 1982. Superoxide dismutase in various tissues from rabbits bearing the Vx-2 carcinoma in the maxillary sinus. Cancer Res. 42, 4233-4235.

Tamura, M., Oshino, N., Chance, B., 1982. Some characteristics of hydrogen- and alkylhydroperoxides metabolizing systems in cardiac tissue. J. Biochem. 92, 10191031.

Underwood, W., Anthony, R., Gwaltney-Brant, S., Poison, A.S.P.C.A., Meyer, R., 2013. AVMA guidelines for the euthanasia of animals: 2013 edition. Schaumburg, IL: American Veterinary Medical Association.

Varju, M., Müller, T., Bokor, Z., Żarski, D., Mézes, M., Balogh, K., 2018. The effects of excessive starvation on antioxidant defence and lipid peroxidation in intensively reared, commercial-size pikeperch (Sander lucioperca L.). Egypt. J. Aquat. Res. 44, 349-352.

Vázquez-Medina, J.P., Crocker, D.E., Forman, H.J., Ortiz, R.M., 2010. Prolonged fasting does not increase oxidative damage or inflammation in postweaned northern elephant seal pups. J. Exp. Biol. 213, 2524-2530.

Vázquez-Medina, J.P., Zenteno-Savín, T., Forman, H.J., Crocker, D.E., Ortiz, R.M., 2011. Prolonged fasting increases glutathione biosynthesis in postweaned northern elephant seals. J. Exp. Biol. 214, 1294-1299.

Wielstra, B., Arntzen, J.W., 2012. Postglacial species displacement in Triturus newts deduced from asymmetrically introgressed mitochondrial DNA and ecological niche models. BMC Evol. Biol. 12, 161.

Wielstra, B., Burke, T., Butlin, R.K., Arntzen, J.W., 2017. A signature of dynamic biogeography: enclaves indicate past species replacement. Proc. Royal Soc. B 284, 20172014

Zhang, Y.K.J., Wu, K.C., Klaassen, C.D., 2013. Genetic activation of nrf2 protects against fasting-induced oxidative stress in livers of mice. PLoS ONE 8, 10. 


\section{Tables}

Table 1. Biometric parameters: snout-vent length (SVL in mm), body mass (BM in g), and condition index (CI) at the beginning and end of experiment for each group. SVL and BM are given as mean \pm standard error of raw data, while the CI was calculated on log-transformed data. F - fasting; $\mathrm{C}$ - control; $\mathrm{N}$ - number of individuals.

\begin{tabular}{|c|c|c|c|c|c|c|c|c|}
\hline & & \multicolumn{2}{|c|}{ SVL } & \multicolumn{2}{|c|}{$\mathrm{BM}$} & \multicolumn{2}{|c|}{ CI } & \multirow[t]{2}{*}{$\mathrm{N}$} \\
\hline & & beginning & end & beginning & end & beginning & end & \\
\hline & $\mathrm{F}$ & $24.49 \pm 0.29$ & $25.59 \pm 0.32$ & $1.22 \pm 0.05$ & $1.08 \pm 0.04$ & $0.245 \pm 0.006$ & $0.221 \pm 0.005$ & 15 \\
\hline \multicolumn{9}{|l|}{ T. macedonicus } \\
\hline & $\mathrm{C}$ & $24.70 \pm 0.48$ & $27.18 \pm 0.58$ & $1.09 \pm 0.05$ & $1.29 \pm 0.06$ & $0.223 \pm 0.010$ & $0.247 \pm 0.006$ & 10 \\
\hline & $\mathrm{F}$ & $25.66 \pm 0.54$ & $26.64 \pm 0.43$ & $1.28 \pm 0.06$ & $1.17 \pm 0.06$ & $0.249 \pm 0.006$ & $0.231 \pm 0.007$ & 13 \\
\hline \multicolumn{9}{|l|}{ T. ivanbureschi } \\
\hline & $\mathrm{C}$ & $25.89 \pm 0.51$ & $28.19 \pm 0.50$ & $1.22 \pm 0.10$ & $1.44 \pm 0.07$ & $0.240 \pm 0.011$ & $0.263 \pm 0.007$ & 8 \\
\hline \multirow{2}{*}{$\begin{array}{l}\text { T. macedonicus } \\
\text { hybrid }\end{array}$} & $\mathrm{F}$ & $27.82 \pm 0.33$ & $28.87 \pm 0.29$ & $1.59 \pm 0.05$ & $1.43 \pm 0.05$ & $0.282 \pm 0.005$ & $0.260 \pm 0.005$ & 15 \\
\hline & $\mathrm{C}$ & $27.85 \pm 0.23$ & $30.19 \pm 0.24$ & $1.59 \pm 0.06$ & $1.72 \pm 0.05$ & $0.281 \pm 0.005$ & $0.290 \pm 0.005$ & 15 \\
\hline \multirow{2}{*}{$\begin{array}{l}\text { T. ivanbureschi } \\
\text { hybrid }\end{array}$} & $\mathrm{F}$ & $23.41 \pm 0.45$ & $24.08 \pm 0.51$ & $0.95 \pm 0.06$ & $0.86 \pm 0.06$ & $0.206 \pm 0.008$ & $0.190 \pm 0.008$ & 13 \\
\hline & $\mathrm{C}$ & $24.69 \pm 0.43$ & $27.06 \pm 0.5$ & $1.01 \pm 0.06$ & $1.18 \pm 0.06$ & $0.214 \pm 0.008$ & $0.233 \pm 0.007$ & 9 \\
\hline
\end{tabular}


Table 2. Results of the comparison between the treatments (fasting and control), "species“ (T. macedonicus, T. ivanbureschi and hybrids) and for the interaction between the treatment $\mathrm{x}$ “species” for biometric parameters (snout-vent length - SVL, body mass - BM and condition index - CI). Data in bold indicate statistical differences.

\begin{tabular}{lllll}
\hline Parameter & Effect & $\mathrm{df}$ & $\mathrm{F}$ & $\mathrm{p}$ \\
\hline \multirow{3}{*}{ SVL-at the beginning } & Treatment & 1 & 2.04 & 0.1565 \\
& Species & 3 & $\mathbf{3 5 . 8 9}$ & $<\mathbf{0 . 0 0 0 1}$ \\
& treatment x species & 3 & 0.76 & 0.5189 \\
\cline { 2 - 5 } BM-at the beginning & Treatment & 1 & 0.57 & 0.4517 \\
& Species & 3 & $\mathbf{3 1 . 3 8}$ & $<\mathbf{0 . 0 0 0 1}$ \\
& treatment x species & 3 & 0.89 & 0.4508 \\
\cline { 2 - 5 } CI-at the beginning & Treatment & 1 & 1.09 & 0.2990 \\
& Species & 3 & $\mathbf{3 0 . 8 4}$ & $<\mathbf{0 . 0 0 0 1}$ \\
& treatment x species & 3 & 1.32 & 0.2745 \\
\cline { 2 - 5 } SVL-at the end & Treatment & 1 & $\mathbf{8 7 . 7 0}$ & $<\mathbf{0 . 0 0 0 1}$ \\
& Species & 3 & 1.62 & 0.1912 \\
BM-at the end & treatment x species & 3 & 1.37 & 0.9371 \\
\cline { 2 - 5 } & Treatment & 1 & $\mathbf{1 4 2 . 9}$ & $<\mathbf{0 . 0 0 0 1}$ \\
& Species & 3 & $\mathbf{3 . 8 2}$ & $\mathbf{0 . 0 1 3 0}$ \\
& treatment x species & 3 & 0.74 & 0.5321 \\
\cline { 2 - 5 } CI-at the end & Treatment & 1 & $\mathbf{4 2 . 6 5}$ & $<\mathbf{0 . 0 0 0 1}$ \\
& Species & 3 & 1.57 & 0.2016 \\
& treatment x species & 3 & 2.50 & 0.0654 \\
\hline
\end{tabular}


Table 3. Results of factorial ANOVA for the comparison between the treatment (fasting and control), “species” (T. macedonicus, T. ivanbureschi and hybrids), and the interaction between the treatment $x$ "species” for oxidative stress parameters. Data in bold indicate statistical differences.

\begin{tabular}{|c|c|c|c|c|}
\hline Parameter & Effect & $\mathrm{df}$ & $\mathrm{F}$ & $\mathrm{p}$ \\
\hline & treatment & 1 & 13.16 & 0.0004 \\
\hline \multirow[t]{3}{*}{ SOD } & Species & 3 & 18.34 & $<0.0001$ \\
\hline & treatment $x$ species & 3 & 6.22 & 0.0006 \\
\hline & treatment & 1 & 27.46 & $<0.0001$ \\
\hline \multirow[t]{3}{*}{ CAT } & Species & 3 & 10.01 & $<0.0001$ \\
\hline & treatment $\mathrm{x}$ species & 3 & 2.38 & 0.0749 \\
\hline & treatment & 1 & 35.06 & $<0.0001$ \\
\hline \multirow[t]{3}{*}{ GSH-Px } & Species & 3 & 13.40 & $<0.0001$ \\
\hline & treatment $\mathrm{x}$ species & 3 & 3.83 & 0.0124 \\
\hline & treatment & 1 & 58.43 & $<0.0001$ \\
\hline \multirow[t]{3}{*}{ GSH } & Species & 3 & 3.24 & 0.0257 \\
\hline & treatment $\mathrm{x}$ species & 3 & 1.35 & 0.2649 \\
\hline & treatment & 1 & 2.03 & 0.1576 \\
\hline \multirow[t]{3}{*}{ GR } & Species & 3 & 3.00 & 0.0344 \\
\hline & treatment $x$ species & 3 & 1.30 & 0.2809 \\
\hline & treatment & 1 & 6.75 & 0.0109 \\
\hline \multirow[t]{3}{*}{ GST } & Species & 3 & 3.55 & 0.0175 \\
\hline & treatment $\mathrm{x}$ species & 3 & 2.16 & 0.0984 \\
\hline & treatment & 1 & 6.63 & 0.0116 \\
\hline \multirow[t]{3}{*}{ SH } & Species & 3 & 25.74 & $<0.0001$ \\
\hline & treatment $\mathrm{x}$ species & 3 & 3.06 & 0.0324 \\
\hline & treatment & 1 & 38.76 & $<0.0001$ \\
\hline \multirow[t]{2}{*}{ LPO } & Species & 3 & 5.61 & 0.0014 \\
\hline & treatment $\mathrm{x}$ species & 3 & 2.76 & 0.0466 \\
\hline
\end{tabular}


Fig.1. Parameters of the AOS (SOD, CAT, GSH-Px and GSH) in fasting and control individuals of parental species (T. macedonicus and T. ivanbureschi) and hybrids (T. macedonicus-mothered and T. ivanbureschimothered). “*” indicates significant differences between treatments (fasting vs control); different letters indicate significant differences between species under fasting treatment. Dot - mean value; box - standard error; bars minimal and maximal value. 

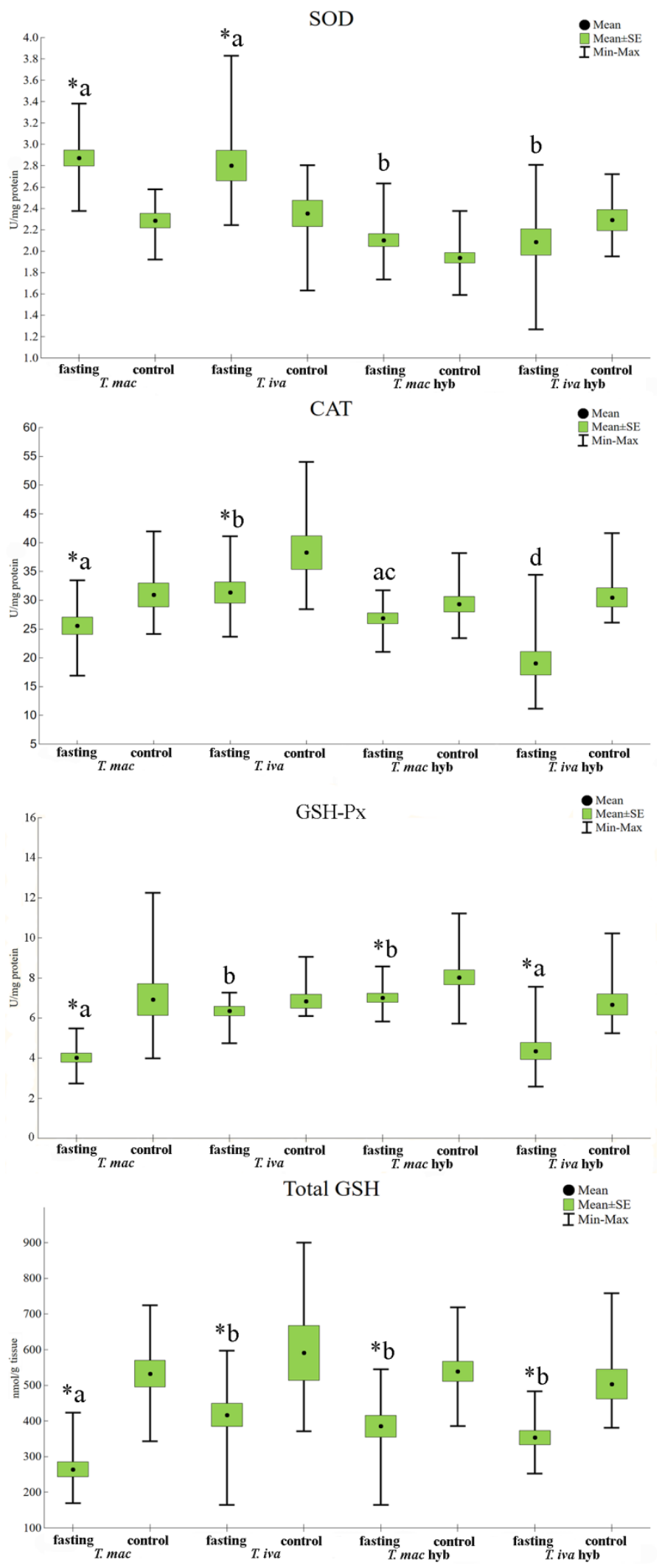

Fig.2. Oxidative stress parameters (GR, GST, SH groups and LPO) in fasting and control individuals of parental species (T. macedonicus and T. ivanbureschi) and hybrids (T. macedonicus-mothered and T. ivanbureschimothered). “*” indicates significant differences between treatments (fasting vs control); different letters indicate significant differences between species under fasting treatment. Dot -mean value; box - standard error; bars minimal and maximal value. 

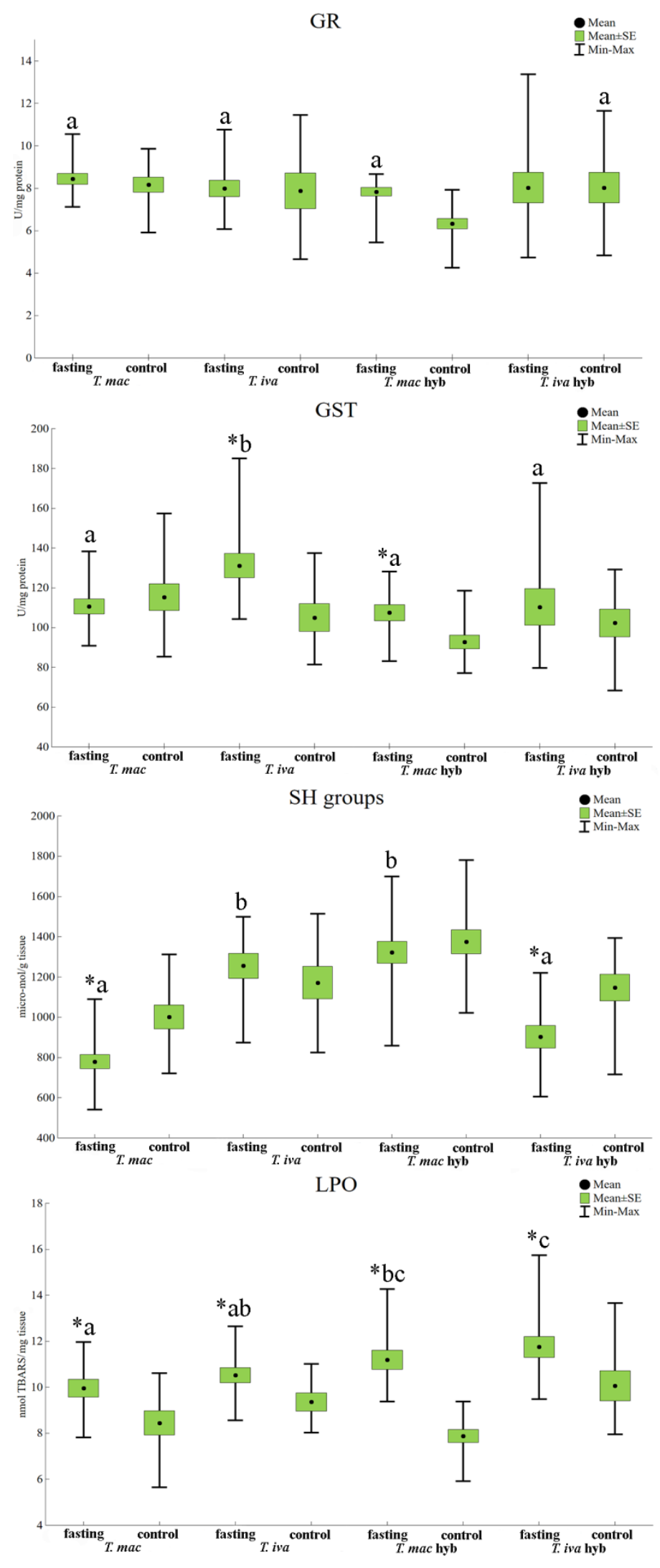